\title{
Sustainability of the IOM 80/20 Nursing Initiative as Viewed through a Decision Support System Supporting "What-If” Benchmarking Capabilities
}

\author{
Christine T. Kovner ${ }^{1}$, Chuo-Hsuan Lee ${ }^{2}$, Edward J. Lusk ${ }^{2}$, Carina Katigbak ${ }^{1} \&$ Nellie Selander ${ }^{1}$ \\ ${ }^{1}$ College of Nursing, New York University, New York, NY, USA \\ ${ }^{2}$ School of Business and Economics, The State University of New York (SUNY) at Plattsburgh, Plattsburgh, NY, \\ USA
}

Correspondence: Edward J. Lusk, School of Business and Economics, The State University of New York (SUNY) at Plattsburgh, Plattsburgh, NY, USA. E-mail: luskej@plattsburgh.edu or Department of Statistics, The Wharton School, University of Pennsylvania, Philadelphia, PA, USA. Tel: 1-518-564-4190. E-mail: lusk@wharton.upenn.edu

Received: January 31, 2013 Accepted: March 27, 2013 Online Published: July 3, 2013

doi:10.5539/jms.v3n3p25 URL: http://dx.doi.org/10.5539/jms.v3n3p25

\begin{abstract}
We present two Decision Support Systems [DSSs] as decision aids to health planners in evaluating the expenditures needed to launch and sustain the $80 / 20$ policy initiative of the IOM. The core DSS is called the Dynamic Change DSS and it is assumed that there is unrestricted activity in the parameter set. The other DSS is called the SWAP DSS; it assumes stasis in the final total number of individuals in the service population and so only a swap between the service classes is permitted. The SWAP is a benchmark to the Dynamic Change information set which is the launching pad to the What-If re-parameterizations that may be needed to address sustainability. The interactive model for these What-If re-parameterizations is an e-Delphi Workspace. These DSSs, the Pilot-Test parameterized versions, and the respective manuals are available from the corresponding author at no charge and with no restriction on their use.
\end{abstract}

Keywords: swapping, dynamic change, sustainability through flexibility

\section{Introduction}

\subsection{Decision Support and the Propagation of Participative Scenario Generation}

The principal advantage of the powerful computing world is that it facilitates the creation of Decision Support Systems (DSSs). DSSs provide informational aids to the Decision Makers (DMs). They are indeed varied and run the gamut from simple data links that provide summaries of data streams connected to Excel ${ }^{\mathrm{TM}}$ or Word ${ }^{\mathrm{TM}}$ display platforms via General User Interfaces (GUI) to integrated interactive networked GUIs that generate expert systems information for the DMs.

A decision-making enhancement often integrated in the DSS functionality is the simple idea of What-If analyses. A What-If analysis, in the DSS context, is the interactive re-parameterization of a DSS from which the DSS informational output is re-calibrated to refine the DMs' understanding of the process under examination; it serves as a useful tool for the DMs to converge the range of possible acceptable predicted event outcomes to a feasible and desirable solution set. Such information is essential to evaluate and argue for sustainability.

DSSs and the related What-If analyses are individually not new concepts; however, their use in combination is just now achieving currency in application. Consider now a few projects that document uses of integrated DSS in generating scenarios for sustainability that enables valuable projects and initiatives to continue. These projects as reported in the literature argue for the need of using integrated DSSs as a forum of interaction to address sustainability issues. They also demonstrate that one of the critical aspects of sustainability is the creation of projections of realistic scenarios that enrich the decision possibility space. The creation of such scenarios, of course, is no guarantee of longevity or sustainability over the planning horizon but we believe that such scenarios enhance the likelihood of sustaining the launched project (Note 1).

The first study to be previewed is Bohner (2006) where the urban planning is examined. Bohner, p.193 notes: 
The question of urban sustainability becomes more and more pressing with regard to the scarcity of urban environmental resources such as ground, air, water, etc. Thus, planners are confronted with the necessity of taking into account impacts typically analyzed by other disciplines. Decision-support systems (DSS) have been developed to introduce multipleinter-disciplinary aspects to the planning process in this complex decision environment. - - -. To illustrate the role of DSS applications in the processes of decision making in today's urban planning, this paper first introduces a select set of approaches to modeling the urban environment and the interaction of actors at different levels of decision making; then a select set of evaluation methods is presented to obtain a more complete simulation of urban dynamics; finally the process of translating the conceptual model of the problem and the decision space into DSS information technology is introduced.

Bohner makes specific mention of selecting a "set of evaluation methods" to obtain a more complete simulation of urban dynamics effected via DSS modeling.

Maia \& Schumann (2007) examined water management in the European Union. They, p.897 note:

Within the Project - 'Developing Strategies for Regulating and Managing Water Resources and Demand in Water Deficient Regions (WSM)' funded by the EU in fifth Research Framework Program, the Ribeiras do Algarve River Basin was chosen as a case study to develop a DSS for planning purposes. - - -. Different ways to improve the water management situation were analyzed: (a) structural options, (b) demand management options and (c) socio-economic measures. These options were analyzed using a range of combinations of extreme demand and availability scenarios and ranked based on indicators reflecting the perception of the local stakeholders towards economic development and social and environmental sustainability. - - -. The application of the DSS follows the following methodology: (a) establishment of the decision context, (b) identification of objectives and criteria, (c) identification of options to solve the problems, (d) estimation of the impacts of the different options and (e) evaluation within a multi-criteria environment.

Maia \& Schumann (2007) also identify the necessity of having an evaluation module-i.e., a benchmark and multiple scenarios as inputs to the decision makers. Based upon the same rationale as Bohner (2006) and Maia \& Schumann (2007), later in our study we will introduce an evaluation benchmark designed to give a perspective on the dynamic projection of the likely scenario space.

Thorburn, Jakku, Webster \& Everingham (2011) report on the use of DSSs as a means of distribution of relevant decision making information among the decision making community. The authors, p. 1473 note:

Decision support systems (DSSs) are one of the ways in which agricultural scientists have attempted to make agricultural systems science more accessible to farmers and to foster innovation. Recently, there has been a shift towards more participatory processes in development and application of DSSs to enhance their end-user use. Apart from increasing adoption, these participatory processes are also likely to enhance co-learning resulting from development/application of DSSs. Learning is a valuable process in increasing sustainability of natural resource management, so the application of DSSs in a learning context can make a contribution to the global challenges faced by agriculture. We developed a framework, using concepts drawn from social studies of science and technology, describing the phases of the participatory DSS development/application process and its likely outcomes. The framework and case study results demonstrate the value of participatory DSS development/application as a co-learning process, an outcome not traditionally valued by agricultural DSS developers and one that is likely to help address the challenges faced by agricultural sustainability.

This idea of DSS distribution and open access is also an aspect that we have embraced in our DSS as we will discuss subsequently.

Finally, and most recently, Koh et al., (2013) articulate and demonstrate the importance of a holistic or integrated understanding of all the possibilities of the various scenarios as they may impact the stakeholders in the system. They, p. 2092 note:

Based upon an increasing academic and business interest in greening the industrial supply chains, this paper establishes the need for a state-of-the-art decision support system for carbon emissions accounting and management, mainly across the product supply chains by...proposing a supply chain framework which provide businesses with a holistic understanding of their supply chains and ensuring partners within supply chain collaborative networks have a shared understanding of their emissions.

Taken together, the aforementioned studies argueclearly that participative involvement, evaluation benchmarking, and multiple scenarios are all needed to realize the information generating impact of DSS needed to focus on sustainability. The evaluation of multiple scenarios can be conveniently made possible through What-If re-parameterizations, which also offer decision makers a means of propagation of experiential learning 
and are relevant in encouraging the development of DSSs in the myriads numbers of fields to which they can enhance the quality of decision making. In this regard, we agree with Newell \& Rosenbloom (1981) whose pioneering work presents convincing arguments that one learns from the varied forms of idiosyncratic applications insofar as the execution of the theory and concepts. Also see: Lee \& Anderson (2001) and Kester, Kirschner\& van Merrienboer (2005).

In this paper, we detail and offer as downloads two DSSs developed to aid health planners in generating information to enhance the quality of the DSS information as it pertains to Institute of Medicine's Committee80/20 Nursing Initiative. In 2010, the IOM Committee in discussing The Future of Nursing proposed to the health care community the need to: "Increase the proportion of nurses with a baccalaureate degree to $80 \%$ by 2020" (Institute of Medicine [IOM], 2010). This initiative is designed to change the constitution of the nursing workforce, and, if implemented, will arrive, after a planning horizon of 10 years, at a workforce of eighty percent ofregistered nurses (RNs) with a Bachelors Degree in Nursing (BSN) and twenty percent with an Associate Degree or a Diploma certification (AD-D). This is a bold and ambitious initiative as according to the results published in 2010 from the 2008 National Sample Survey of Registered Nurses by the Health Resources and Services Administration (HRSA, 2010), only $34.2 \%$ of RNs in the United States have a Bachelor's degree or higher as their initial nursing degree, whereas $65.8 \%$ have an Associate's degree or a Diploma. Clearly, such a proposal to re-orient the constitution of the nursing workforce will challenge the Nursing Education System (NES), and its implementation would require macro-system transformation that involve a variety of stakeholder groups in the decision making process.

Specifically, this paper responds to the challenge of the IOM Initiative. By offering two decision support systems (DSSs) that integrate different factors and allow participants to maneuver with dynamism, we provide a malleable modeling context to help decision makers address two questions raised by the IOM initiative: (1) Is The 80/20 Initiative feasible? (2) And, if so, what are the expenditure consequences of achieving such a dramatic reorganization of the RN workforce? These two DSSs are intended to alleviate the information overload and the poorly organized information that often accompanies macro-projects that may hinder DMs from efficiently evaluating the temporal and long- term sustainability of the IOM Initiative.

In the following pages, we will:

1.) Present and discuss two decision support systems: a SWAP: DSS and a Dynamic Change: DSS, which were developed to assist healthcare workforce planners in evaluating the expenditures required to achieve The 80/20 Initiative. The SWAP: DSS assumes that: (1) the total nursing workforce remains the same over the planning horizon, and (2) the number of BSN RNs needed are achieved as a direct reduction of the number of AD-D RNs; whereas the Dynamic Change: DSS assumes that the BSN and AD-D workforces will be changing over the planning horizon and computes the number of additional BSN RNs that will be needed to achieve The 80/20 Initiative.

2.) Offer a novel approach of benchmarking the planning information generated by the Dynamic Change: DSS by using the SWAP: DSS results. We selected the Swapping concept as a benchmark because mathematically, swapping requires the fewest number of conversions-AD-D to BSN RNs-and so will be the lowest expenditure alternative. Therefore, the Swap number will serve as a good relative benchmark to evaluate proposals made by the DM to achieve The 80/20 Initiative.

3.) Illustrate the parameterization of the SWAP and Dynamic Change DSSs using information from selected pilot tests.

4.) Discuss the What-If concept and the extension to distributed decision information as a means of participative convergence to an action plan employing a Delphi Model. This is the enriched set of decision possibilities that allow decision makers to consider all the various aspects of the project and thus be sensitive to selecting decisions that allow for project sustainability in the planning horizon.

\section{Method}

\subsection{The DSSs for Evaluating the 80/20 Initiative}

Generating information to determine the feasibility and expenditure consequence of achieving The 80/20 Initiative is one of the pre-conditions for effective planning given the complicated nature of a systemic change of this magnitude. We have identified economic feasibility as the measure that impacts sustainability in the planning horizon. The argument is a simple one in the nursing educational milieu. As indicated above, moving from a work environment where about one-third of the nursing workforce has a BSN to where $80 \%$ have such an academic degree in a ten year time frame is a challenging macro project. Without a modeling perspective to 
examine the various expenditure consequences of proposed action plans, DMs would be forced to confront the risk of embarking upon a program that has intellectual appeal but has not been considered carefully in terms of its expenditure consequences. The great tragedy is that after initial resources are committed early in the project for a few years the DMs only to find that the resource commitment cannot be sustained at a level to realize the project; often then the project is not realized and many of the resources committed at the launch may be wasted. This, for us, is the sustainability issue and so we are focused on examining the expenditure issues before decisions are made to launch.

To generate such information and to provide DMs with sufficient flexibility to test various What-If assumptions so as to profile the expenditure consequences, we created the following two DSSs-SWAP and Dynamic Change. Each of these DSSs includes four principal worksheet modules with each worksheet module organized around the concept of decomposition-i.e., where the various important elements of the information set for the particular worksheet are indicated and the DM is prompted to enter this elemental information; then the information is aggregated and transferred to the next worksheet. Consider now the structure of these four modules.

1.) Worksheet I: Baseline Workforce Information. There needs to be a simple way for a DM at the national, state, regional, county, or city level - i.e., the location context - to develop baseline information regarding the number of BSN RNs and AD-D RNs currently in the nursing workforce. The DM is asked to provide data as defined following and then inputs this data in the appropriate color-coded "Input Cells" of the DSS. It is critical to understand that the numbers inputted are estimates using the best available data, such as adjustments to the last year of reported information or three year averages. Typically, for example, the DM will collect this information from reported information sources such as the National Council of State Boards of Nursing, State Boards of Nursing, and/or Special Surveys. The elemental Baseline inputs are:

- Number of RNs with a BSN or higher currently in the workforce

- $\quad$ Number of RNs with less than a BSN currently in the workforce (e.g. diploma, AD-D)

- Number of BSN-prepared RNs added to the workforce by examination

- Number of AD-D RNs added to the workforce by examination

- Number of BSN-prepared RNs added to the workforce as international graduates

- Number of AD-D RNs added to the workforce as international graduates

- Number of BSN-prepared RNs added to the workforce as state transfers

- Number of AD-D RNs added to the workforce as state transfers

- Number of BSN-prepared RNs that left the workforce (includes non-renewals, retired, temporary leave, and transfers out of state and deaths)

- Number of AD-D RNs that left the workforce (includes non-renewals, retired, temporary leave, and transfers out of state and deaths)

- $\quad$ Number of RNs converted from AD-D to BSN-prepared (RNs added to the BSN workforce)

- $\quad$ Number of RNs converted from AD-D to BSN-prepared (RNs leaving AD-D workforce)

This data is summarized and then transferred to "Worksheet II: Required Number of BSN Needed Over What Would Occur Normally."

2.) Worksheet II: Required Number of BSN Needed Over What Would Occur Normally —based upon historical projections. This is the most critical worksheet in the DSS system. Recall that there are two different concepts under which this required number of BSN is developed. One is the Dynamic Change: DSS and the other is its relative benchmark: the SWAP: DSS. Following we will consider parameterization of the Dynamic Change: DSS as it is the projection of what the DM feels will happen over the planning horizon. Based upon the current workforce status information developed in Worksheet $I$ above, the DM will input:

a. The number of years until the end of the planning horizon;

b. The expected change in the number of BSN andAD-D RNs over the planning horizon given a specific set of policy initiatives likely to take effect during the planning horizon, the sum total of which are likely to impact the number of RNs educated at various levels during the planning horizon;

c. The number of years before any policy initiatives are likely to affect the NES; 
d. The number of qualified BSN and AD-D RNs that can be expected to enter the workforce during the period of time both before and after the initiatives take effect; and

e. The number of RNs that are expected to leave the workforce during the planning horizon for all the various reasons, such as: retirement, transfer to other professional positions, and death.

These various inputs permit the calculation of the number of BSN RNs needed to be added to the BSN RN workforce by the end of the planning horizon to achieve The 80/20 Initiative. Then, this information is transferred to Worksheet III.

3.) Worksheet III: Feasibility. This is the first issue related to sustainability: Feasibility as a feature of the Nursing Education System (NES). Given the number of BSN RNs that need to be produced by the NES to achieve The 80/20 Initiative, either under the SWAP or the Dynamic Change contexts, the systemic feasibility of adding this required number of BSN RNs will be assessed in this worksheet module. This assessment will require the DM to use the numbers from Worksheet II to estimate the following elements:

a. The number of BSN RNs that currently graduate from select types of nursing education programs in the location context that the DM is analyzing, and

b. The possible increase in such BSN RNs graduates given assessments of the NES resources in the location context.

Considering the total number of BSN RNs that could feasibly be produced given the NES configuration in the location context, the DSS determines if a shortfall in the number of BSN-prepared RNs needed would likely result. If there is such a shortfall, then the DM will indicate how many BSN RNs from other countries-i.e., an international contingent of RNs - could be used to fill the shortfall gap. If this gap cannot be filled, then this shortfall raises the first sustainability red-flag. By definition if the initial goal is not feasible the DM group may want to re-address the espoused goal to arrive at a second-best feasible goal among all available options.

4.) Worksheet IV: Expenditure Consequence of the 80/20 Policy. In the practical context within which this Dynamic Change: DSS will be used, the focus is on the price to be charged-i.e., the Expenditure needed to achieve The 80/20 Initiative. Then the DM will be asked to enter:

a. The estimated current price of tuition for each of the Program Groups-e.g., generic nursing programs broken down by Private and Public institutions in the NES;

b. The related costs, such as books and fees; and

c. The number of years to complete each targeted academic program, and the estimated inflation and general percentage increase/decrease per year expected for each program type.

This expenditure basis may also be viewed as the cost/resource commitment agencies must make to create the individual policies necessary to realize The $80 / 20$ Initiative. This is the second issue related to sustainability: securing the funding to uphold the project. Given this final determination of the number of BSN RNs needed, as developed in the previous Feasibility section of the DSS, the practical expenditure expectation of achieving the desired ratio of BSN to AD-D RNs, can be computed. Then, after reviewing the above practical expenditure figure the DM may be prompted to apply the DSS to explore the possibilities of achieving other possible feasible and reasonable numbers of BSN RNs with different levels of cost/resource commitment. In addition, the DSS offers an application (APP) that estimates the incremental costs of meeting different project goals. This incremtndal cost-APP is based upon the sets of policy initiatives that may restrict funding to support only those individuals who of their own volition have taken the educational-tract and have an AD-D at the time of applying for grant extension to continue their studies. The incremental cost may be a "fallback" calculation in that the resource commitment will by definition be less than the fully-costed perspective that we have assumed for the IOM initiative. This will be the final computational stage of either the SWAP: DSS or the Dynamic Change: DSS.

\section{Results}

\subsection{Illustration of the Two DSSs Using the Pilot Test Information}

We will now illustrate, using pilot test information, the functionality of the two DSSs. After we consider the "operational" nature of the Dynamic Change: DSS and its benchmark the SWAP: DSS, we will move to the macro-decision-making environment within which the various What-If profiles will be used to make the two sustainability decisions, including Systemic Feasibility of the NES and the likelihood or probability of garnering the resources needed to effect the project— the Committed Expenditure Funding. The SWAP: DSS has fewer parameters and it is simpler to present without loss of generalizability regardingthe overall functionality of the 
DSSs. Therefore, all of the information to be used in our illustration will come from the SWAP pilot-test. After our illustration of the SWAP: DSS we will then present selected aspects of the Dynamic Change: DSS and compare the Dynamic Change: DSS with SWAP: DSS benchmark to demonstrate the advantages of using both DSSs to the end of generating a holistic profile set that will offer the trade-offs to enable sustainability.

\subsection{The SWAP: DSS Benchmark}

From one of the pilot tests, the number of BSN and AD-D RNs estimated at the baseline year using Worksheet I were: 152,660 and 124,178 respectively. This gives, under the stasis assumption of the SWAP: DSS, a total workforce at 2012 and also at 2021 (the end of the ten year planning horizon) of: 276,838 [152,660 + 124,178]. This information was transferred to Worksheet II: The Number of BSN RNs Needed to be Swapped. The BSN-ratio of interest in 2012 is:

$$
B S N /[B S N+A D-D]=\text { Percentage of BSN RNs at Baseline }
$$

or

$$
152,660 /[152,660+124,178]=55.14 \%
$$

As the policy goal is to reach $80 \%$ by 2021 , the value of the short-fall, $\delta$, will be: $\delta=0.80-0.5514=0.2486$. Therefore, according to Eq 7 (see Kovner, Lee, Lusk, Katigbak, \& Selander (2013), the number of BSN nurses to be swapped, $\mathrm{B}^{\Delta}$, is:

$$
B^{4}=\delta \times[B+A]=0.2486 \times[152,660+124,178]
$$

or

$B^{\Delta}=68,810$

The demonstration proof of this calculation is:

$[152,660+68,810] /[(152,660+68,810)+(124,178-68,810)]=80 \%$

The above calculation shows that over the ten-year planning horizon there must be a reduction of the number of AD-D RNs and an exact increase of BSN RNs-i.e., a SWAP of 68,810 RNs. That is to say, to meet The 80/20 Initiative there must be 68,810 new BSN RNs added to that workforce for arriving at 221,470 BSN RNs where the total workforce is assumed to remain the same as it was at the baseline year-i.e., stasis of 276,838 . Underlying this SWAP context is the assumption that the policy makers will be able to maintain extensive and relatively absolute control of the NES via direct or indirect legislation or incentive programs. This mathematically derived swap information-i.e., $68,810 \mathrm{BSN}$ is then transferred to Worksheet III: Feasibility. This worksheet starts the examination of the sustainability of the project-specifically the feasibility component. The DM enters in Worksheet III various estimates that will be used to calculate the number of BSN RNs that will be needed over what would be expected to achieve the planning goal. For these parameters, the pilot tester assumed that:(1) it would take two years for the SWAP-initiatives to take place, (2) the annual BSN RN output of the NES would be 4,525, and (3) finally that the number of BSN RNs leaving the workforce annually would be $4.7 \%$. With these parameters entered the DSS calculates that 7,838: $[[68,810-(2 \times 4,525)] /(1-.047)] /(10-2)$ new BSN RNs need to be added to the BSN RN workforce and so an equal reduction of the number of AD-D RNs each year need to be made to realize The 80/20 Initiative. The DM pilot tester estimated that on an annual basis during the planning horizon the NES can generate 4,525 BSN RNs, leaving a shortfall of 3,313 BSN RNs annually $[7,838-4,525]$. Next, the DM is asked to estimate the increase in BSN RN production that could be expected if the slack in the NES were to be utilized-i.e., reduced. The pilot tester assumed that there would be a realistic possibility to increase the BSN RN production by 1,748 through better unitization of the NES resources, thereby reducing the annual shortfall to 1,565 [3,313 - 1,748]. At this point, the only additional source of BSN RNs is to add them from the pool of internationally educated BSN RNs. Let us further assume that given all the policy implications of green-card approvals, the DM believes that 1,025 international BSN RNs can be counted on annually to join the nursing workforce in the location context under examination. In this case, there will be a slight final annual shortfall of 540 [1,565-1,025] BSN-prepared RNs. Looking at the comparative totals over the planning horizon the pilot tester needed a total of 221,470 BSNs to achieve the $80 / 20$ policy goal; after considering the feasibility of all the possibilities, the DSS arrives at $217,150[221,470-540 \times(10-2)]$ as a feasible number of BSNs. Therefore, the $80 / 20$ ratio is almost satisfied; the shortfall is very small relative to the total. For example, the actual ratio is: $217,150 / 276,838=78 \%$

Therefore, in this case the actual realistic expectation for production of BSN RNs is $64,490[68,810$ - the total shortfall of $540 \times 8=4,320]$ or $[68,810-4,320]]$. Here is the first sustainability decision. Can the DM accept the modified goal as it does not look as though the exact swaps can be made to achieve the 80/20 Initiative? With 
this modified goal which is feasible and sustainable in the first measure- $78 \%$ rather than $80 \%$ the DM will then proceed to the final stage of the DSS, Worksheet IV: The Expenditure Consequence Worksheet. This is the last of the four sections of the DSS. Here, the DM is asked to determine the various educational programs that could be used to generate the required number of additional BSN RNs as well as the tuition and related expenditures associated with each program. Fixing these parameters, and assuming that the number of BSN RNs required to be generated was 64,490 the DSS produces the following table which displays the full $100 \%$ expected expenditures. In addition, the expenditures for achieving only $2 / 3$ and $1 / 3$ of the $64,490 \mathrm{BSN}$ RNs modified goal are reported as a relative benchmark comparison. This additional information we feel will encourage the DM to consider the various levels of expenditure in relation to the resources they need to garner to fulfill their commitment.

Table 1. Final output of the cost consequence worksheet based on the SWAP: DSS

\begin{tabular}{llll}
\hline & $100 \%$ & $67 \%$ & $33 \%$ \\
\hline Number of BSN Students & 64,490 & 43,208 & 21,289 \\
Total Tuition & $\$ 3,538,977,517$ & $\$ 2,371,114,937$ & $\$ 1,167,862,581$ \\
Total Related Expenditures & $\$ 359,687,539$ & $\$ 240,990,651$ & $\$ 118,696,888$ \\
Total Projected Expenditures & $\$ 3,898,665,056$ & $\$ 2,612,105,587$ & $\$ 1,286,559,468$ \\
\hline
\end{tabular}

Recall that the purpose of the SWAP: DSS is to provide a focused and appropriate benchmark to the expenditures expected under the Dynamic Change: DSS. Now, for simplicity we will apply the same baseline information in the Worksheet I of the SWAP: DSS to the more complicated context of the Dynamic Change: DSS.

\subsection{Benchmarking: The Dynamic Change: DSS relative to the SWAP: DSS}

We will now provide an example of the Dynamic Change context using the same baseline information as reported above for the SWAP: DSS illustration. Recall that the numbers of BSN and AD-D RNs estimated at the baseline year of 2012 are 152,660 and 124,178, respectively. Assume now for the Dynamic Change context that the total numbers of BSN and AD-D RNs in the workforce are both projected to grow by $5 \%$ annually. In this case, the number of BSN RNs needed to achieve the $80 / 20$ policy goal given the Dynamic Change context would be 560,424 BSN RNs! (Note 2). Here the pilot tester decided to pass over the feasibility computation [Worksheet III] as it was previously determined that under the swap conditions less than 68,810 could be expected to be created by swapping. To expect that 560,424 BSNs on net could be created given the current NES even counting the slack in the system was judged to be highly improbable. But nonetheless it is instructive to determine the expenditure consequence for such an improbable outcome as a means of ascertaining a context for determining the nature of the project that could be feasible and sustainable. Therefore, continuing with this example, the DM allocated these BSNs uniformly over the same programs that we used in the SWAP example above, would result in expenditures as follows:

Table 2. Final output of the cost consequence assuming 5\% growth for both of the BSN and AD-D RNs based on dynamic change: DSS

\begin{tabular}{llll}
\hline & $100 \%$ & $67 \%$ & $33 \%$ \\
\hline Number of BSN Students & 560,424 & 375,484 & 184,940 \\
Total Tuition & $\$ 38,667,495,509$ & $\$ 23,897,221,991$ & $\$ 11,770,273,518$ \\
Total Related Expenditures & $\$ 3,008,436,257$ & $\$ 2,015,652,292$ & $\$ 992,783,965$ \\
Total Projected Expenditures & $\$ 38,675,931,766$ & $\$ 25,912,874,283$ & $\$ 12,763,057,483$ \\
\hline
\end{tabular}

Here we can clearly see the effect of not having the authority, ability or willingness to effect the necessary changes in the NES to expeditiously reach The 80/20 Initiative by swapping. This flexibility is a critical aspect of sustaining the possibility of realizing the project. If one simply lets the BSN and the AD-D RN workforces grow at the same rate (5\% per year), at the end of the planning horizon the number of BSN RNs needed to reach the policy goal will require expenditures of $\$ 38.7$ billion. As a benchmark against the SWAP: DSS, which is effected under the assumption of stasis and relative control and using a slight reduction in achieving the policy goal: 64,490 relative to 68,810 of the NES, the Expenditure Benchmark Multiplier (EBM) can be calculated as follows: 
or

$E B M: 9.92=\$ 38,675,931,776 / \$ 3,898,665,056$

This EBM will always be greater than 1.0, even when the AD-D RN workforce is unchanged over the planning horizon, as demonstrated in Kovner et al., (2013). In this case, the EBM simply indicates that about ten (10) times the resources will need to be expended unless policy can control the relative NES components of the BSN and AD-D RN workforces so that the AD-D workforce is swapped. We suggest that the EBM is a critical ratio, as it represents the resource trade-off for opting to not move into a SWAP environment when it is clear that the BSN and AD-D constituencies have a tendency to increase.

\subsection{What-if Analyses: Scenario Modification through the Delphi Process}

The Delphi Process is the model by which logistical communication interactions are organized. In this way the Delphi Process is essentially a commitment by the participating DMs to engage in the continuous process of creating information from data, and distributingthe information to other DMs, who in turn will retrieve, improve, and share,often as feedback, the information with the DMs involved in the process. At some point the scenario or project modifications are minimal and the information is therefore agreed upon and this information set is used to make the required decision. Essentially, then a Delphi Process modelling logistic is a course of action involving convergent and interactive decision making. We offer this as the model for the DSS What-If scenario regenerations. Also, we prefer the EDI-mode (Jung-Erceg, Pandza, Armbruster \& Dreher, 2007) where the DMs discuss, in a Chat-Loop-Context, the various outputs of the DSS What-If until they are satisfied with their insights-i.e., an e-Delphi Process. This DSS-What-If-e-Delphi process ensures that all of the identified DM will have the possibility to create their DSS scenario modification that will be available to all the other DMs in the process. Given that during the What-If e-Delphi interchanges and modifications the DMs will observe the Feasibility and Expenditure consequences of the various benchmarked projections, they will be sensitized to the practical projected realities; this should enhance the convergence to a project launch that has been fine tuned to be sensitive to sustainability. In fact this is exactly what the above cited literature suggests as the benefit of interactive use of DSSs. Consider now a normative illustration of this Delphi process:

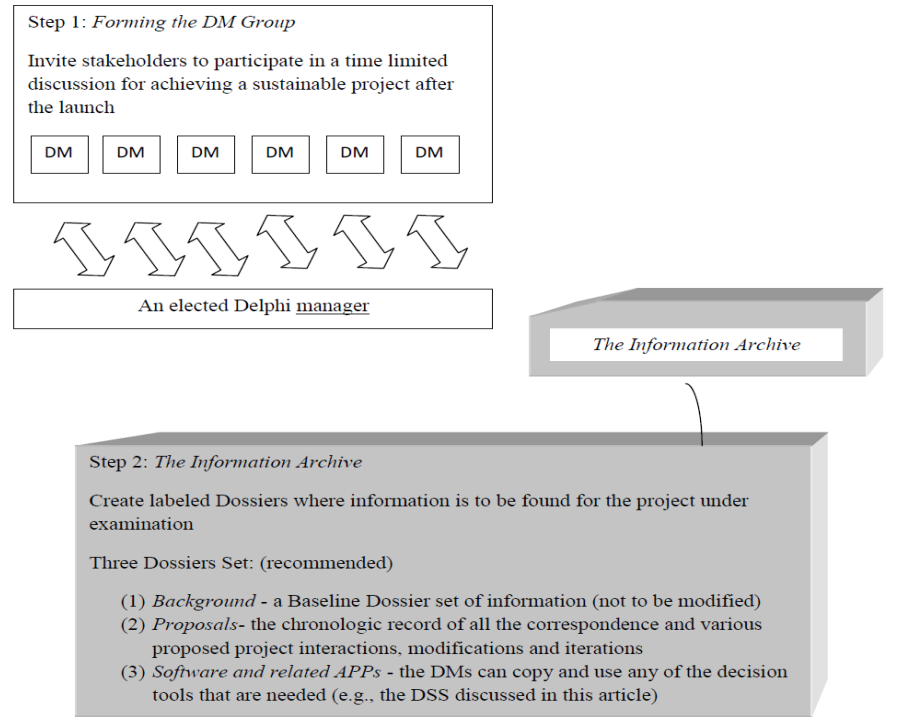

Figure 1. Creation of e-delphi communication space

\subsection{Creation of the e-Delphi Communication Space}

Using the various e-links that exist, such as $\mathrm{Skype}^{\mathrm{TM}}$, chat-rooms, posting spaces, to mention a few, the following three general steps, also scripted out in Figure 1, are recommended:

1.) Forming the DM Group Invite stakeholders to participate in a time limited discussion for achieving a sustainable project. We offer that sustainability is linked to achieving the project after the launch during the 
planning horizon of the project. Sustainability as a "forever" concept often creates an impediment to decisions that have a temporal reality.

2.) The Information Archive Create labeled dossiers where information is to be found for the project under examination. We recommend three dossiers sets: Background, Proposals, and Software and related APPs. The Background Dossier has, of course, many sub-dossiers where various aspects are contained such as a sub-dossier for the Grant Prospectus containing all of the specifications of the grant, and a sub-dossier for PDF readings germane to the grant project that address feasibility as well as sustainability issues. We recommend this information not to be modified - i.e., it is a Baseline Dossier set of information. The Proposal Dossier has the chronologic record of all the correspondence and various proposed project interactions, modifications and iterations. Each new modification, regardless of the source of information, will be posted in a dossier labeled as to time of the posting of the suggested modified project. This archive will continue to grow until the project is resolved. Finally, there is a Software and APP Dossier space. Here the DMs can copy and use any of the decision tools that are needed. For example, the DSSs discussed previously will be part of this dossier.

3.) Often there is an elected Delphi manager who will have chat-links such as those found on Facebook ${ }^{\mathrm{TM}}$ or Twitter ${ }^{\mathrm{TM}}$ to keep the DMs apprised of what is transpiring and may of course encourage participation. Often this person is the principal investigator (PI) of the grant. This sort of friendly guidance is needed as the e-Delphi Process is time limited.

Following we present an illustrative example of the modification process. Consider the illustration that we provided above where the initial parameterization of the DSS isolated two impediments to sustainability:

1.) The SWAP proposal where 68,810 BSNs needed to be created through swapping could not exactly be achieved; the expenditures of swapping to produce 64,490 BSNs were about 3.9 billion dollars. This was judged to be possible, certainly not a guarantee but "in the expenditure sustainability ballpark."

2.) However, the Swap-profile required a degree of centralized decision making control over the NES that was judged to be unrealistic. In this case the SWAP proposal was "in the expenditure sustainability ballpark" but not likely to be sustainable from a decentralized political context. In order to adapt the analysis to a decentralized political environment, the DM would weigh the option to relax the requirement of exercising political control over the NES and as a result would turn to the Dynamic Change DSS. Herein, both the BSN and AD-D nursing populations were judged to grow by $5 \%$ over the planning horizon. Therefore, this eliminated the sustainability issue of political control of the NES, but to do so required about a 10 fold increase in the number of BSNs to produce over 500,000 additional BSNs; this figure was judged to be not systemically feasible. Also, an expenditure of around 38.9 billion for supporting the 500,000 additional BSNs was judged to be impossible in the current funding climate.

In this case the DM is faced with a sustainability conundrum. The Number of BSNs through the SWAP is not feasible; however, the expenditure of 3.9 billion was judged to be possible over a ten year planning horizon. The Dynamic Change effected under an assumption that the AD-D and the BSN RNs both grow at 5\% per year produces a result that is practically impossible from a feasibility and expenditure perspective. This conundrum initiates the Delphi Process.

In the Delphi mode, various DMs are invited to participate and given access to the SWAP and the Dynamic Change Tables as well as the two DSSs. With this information and an understanding of the goal- to achieve the $80 / 20$ IOM initiative - they will generate the next round of What-If alternatives that will be uploaded for subsequent examination by the invited members of the DM group in the e-Delphi context. This process of inspection, What-If modification, and re-modification will continue until there is consensus that (i) the 80/20 IOM project cannot be expected to achieve sustainability-i.e., to be realized, as is, in the planning horizon or (ii) the modifications are agreed to by the DM group and the modified project is expected to achieve sustainability over the planning horizon and so merits consideration to be launched (Note 3). Consider now an example of the modification and information generated as an illustration of the e-Delphi Process.

\subsection{Recommendede-Delphi Protocol}

The outputs of the initial parameterizations, Table 1 and Table 2, are uploaded to the e-Delphi-Workspace. The invited DMs are free to download them, use the DSSs, and create various What-If modifications that are uploaded to the temporally labeled dossier to offer other possible scenarios. These and subsequent interactions among the DMs serve the goal which is to develop a final decision that would address sustainability in Feasibility and Expenditure consequence. 
For example, one of the individuals in the invited DM group may believe that the projected expenditures of $\$ 38$ billion and the need to producealmost 500,000 additional BSNs, as illustrated by the Dynamic Change DSS, cannot possibly be funded considering that governments are unable to fully fund education budgets given current economic conditions. She therefore turned to the SWAP model which places about one-tenth the demands on the NES. Using the SWAP: DSS the cost per BSN is computed where various educational programs were used to create the $64,490 \mathrm{BSNs}$; this cost was $\$ 60,454$ per student. Inspired by above cost figure of the SWAP: DSS, she believes that a "tuition and related cost 100\% reimbursement program" offered to qualified high school on the condition that they take a four year BSN On-line Educational Program could attract sufficient numbers to act as an tacit-swap-it would achieve the same goals with no central or political administrative control of producing the 68,810 nurses needed to achieve the IOM $80 / 20$ goal.

In this case the What-If output is obtained by assuming that the SWAP context could be realized by drawing students that would otherwise go to AD-D programs to accept the full educational support for On-Line programs which are the least cost alternative identified by the DM regarding the NES. Again, using the DSS she finds that the cost per student for the On-Line option is $\$ 36,794$ as opposed to $\$ 60,454$. She argued that a small portion of this savings of 1.5 billion $[64,490 \times(\$ 60,454-\$ 36,794)]$ could be made available to increase the capacity of the NES so as to realize the additional BSN needed over the planning horizon. Further, she argues that 500 million, a third of the projected savings, would be needed to expand the NES of the ten year planning horizon to achieve the IOM goal. Therefore, this What-If re-parameterization will be uploaded to the Delphi Space as:

\section{Detailed Computations and Results using the SWAP: DSS}

Tuition \& Related Costs: $\$ 36,794$ per BSN student. Given the expansion of the NES, the goal of achieving the 80/20 policy by producing 68,810 BSNs can become feasible when the Contributed Costs to expand the NEC are $\$ 500,000,000$.

\section{Total Expenditure Consequence:}

Tuition and Related Costs: $\$ 2,531,862,758$ [ $\$ 36,794$ per BSN student x 68,810 BSNs]

Contribution to the NEC Expansion $\$ 500,000,000$

Total $\$ \mathbf{3 3 , 0 3 1 , 8 6 2 , 7 5 8}[\$ 2,531,862,758+\$ 500,000,000]$

Referencing Table 1, the above total of $\mathbf{\$ 3 , 0 3 1 , 8 6 2 , 7 5 8}$ is compared to the SWAP cost of achieving 64,490 BSNs using a variety of NES programs: $\mathbf{\$ 3 , 8 9 8 , 6 6 5 , 0 5 6}$.

This What-If re-parameterization of the SWAP DSS shows that the $80 / 20$ goal would become feasible and the issue of sustainability can be addressed as the SWAP DSS result indicates that this modified scenario requires fewer resources than the initial SWAP result, resulting in an additional savings of:

$\mathbf{\$ 8 6 6 , 8 0 2 , 2 9 8}[\$ 3,898,665,056$ - $\mathbf{\$ 3 , 0 3 1 , 8 6 2 , 7 5 8 ]}$ that could be used to provide subvention to encourage students to take the On-line option.

Figure 2. What-If assumption: focus on the SWAP result and on-line programs

This particular What-If re-parameterization would then be viewed by others in the DM group and similarly other members would upload their possibly adapted information based on the assumptions of their own scenarios. Aftera period of time, this interaction process among members will have a variety of options converged to a limited number of options, and ultimately the final solution will be determined by the DM. Here, we recommend the DM to allow only a definitive time frame for What-If interactions; otherwise, we find that closure is often a problem.

\section{Discussion and Conclusion}

We offer two Decision Support Systems as valuable tools in the Decision Maker's panoply. The SWAP: DSS serves as a benchmark; it assumes that (i) there will be stasis with respect to the size of the total nursing workforce, and (ii) the increase in the number of BSN RNs needed to satisfy The 80/20 Initiative occurs as a 1-to-1 reduction of the number of AD-D nurses. In contrast, the Dynamic Change: DSS relaxes the SWAP assumptions and allows change in the BSN and AD-D RN workforces, and so in the total nursing workforce. These two DSSs, programmed in Excel ${ }^{\mathrm{TM}}$, can be used by the DM to determine: (1) the number of BSN RNs 
needed to be added to the workforce by the end of the planning period to satisfy The $80 / 20$ Initiative, (2) the systemic feasibility of adding this number of BSN RNs, and (3) the expenditure consequence of realizing The $80 / 20$ Initiative. We then recommend that the initial parameterizations of these DSS be uploaded to an e-Delphi Workspace and a group of decision makers be invited to engage in systemic re-parameterizations to address the Feasibility and/or the Expenditure Consequence issues that may compromise sustainability of the project in the planning horizon. All of this DSS information and the various What-If re-profiling are necessary if the DM is to arrive at a reasoned understanding of the temporal and so the long-term possibilities of sustaining the launched IOM initiative. These DSSs, the Pilot-Test parameterized versions, and the respective manuals (Kovner, Katigbak, \& Selander (2013a; 2013b) are available from the corresponding author upon request; we waive all intellectual property rights to their use.

\section{Acknowledgements}

This project was enabled by the generous support of The Robert Wood Johnson Foundation: Grant: RWJF: 4466(2011), Principal Investigator: Dr.Kovner: ctk1@nyu.edu. We thank the number of nursing professionals who provided valuable pilot-test feedback, in particular New York State Board of Nursing Executive Secretary, Barbara Zittel, PhD, RN, New Mexico Center for Nursing Excellence Executive Director, Pat Boyle, MSN, RN and University of New Mexico College of Nursing Professor and Executive Dean, Jean Giddens, PhD, RN, FAAN.

\section{References}

Bohner, C. (2006). Decision-support systems for sustainable urban planning. International Journal of Environmental Technology and Management, 6, 193-205. http://dx.doi.org/10.1504/IJETM.2006.008261

Health Resources and Services Administration, U.S. Department of Health and Human Services. (2010). The Registered Nurse Population: Findings from the 2008 National Sample Survey of Registered Nurses. Retrieved from http://bhpr.hrsa.gov/healthworkforce/allreports.html

Instituteof Medicne [IOM] . (2010). The Future of Nursing: Leading Change, Advancing Health. Retrieved from http://books.nap.edu/openbook.php?record_id=12956\&page=R1

Jung-Erceg, P., Pandza, K., Armbruster, A., \& Dreher, C. (2007). Absorptive Capacity in European Manufacturing: A Delphi study. Industrial Management and Data Systems, 107(1), 37-51. http://dx.doi.org/10.1108/02635570710719043

Kester, L., Kirschner, P. A., \& van Merrienboer, J. J. G. (2005). The Management of Cognitive Load during Complex Cognitive Skill Acquisition by Means of Computer-Simulated Problem Solving. British Journal of Educational Psychology, 75(1), 71-85. http://dx.doi.org/10.1348/000709904X19254

Koh, S. C., Genovese, A., Acquaye, A. A., Barratt, P., Rana, N., Kuylenstierna, J., \& Gibbs, D. (2013). Decarbonizing product supply chains: design and development of an integrated evidence-based decision support system - the supply chain environmental analysis tool. International Journal of Production Research, 5l(7), 2092-2109. http://dx.doi.org/10.1080/00207543.2012.705042

Kovner, C., Katigbak, C., \& Selander, N. (2013a). Manual for SWAP: Decision Support System.

Kovner, C., Katigbak, C., \& Selander, N. (2013b). Manual for Dynamic Change: Decision Support System.

Kovner, C., Lee, C-H., Lusk, E., Katigbak, C., \& Selander, N. (2013). The Technical Underpinnings and Extended What-If Analyses of the Decision Support Systems Programmed for the IOM 80/20 Nursing Initiative. Working Paper. SUNY: Acctg2013.

Lee, F. J., \& Anderson, J. R. (2001). Does Learning a Complex Task Have to be Complex? A Study in Learning Decomposition. Cognitive Psychology, 42(3), 267-316. http://dx.doi.org/10.1006/cogp.2000.0747

Maia, R., \& Schumann, A. H. (2007). DSS Application to the Development of Water Management Strategies in Ribeiras do Algarve River Basin. Water Resource Management, 21, 897-907. http://dx.doi.org/10.1007/s11269-006-9106-3

Newell, A., \& Rosenbloom, P. S. (1981). Mechanisms of Skill Acquisition and the Lawof Practice. In J. R. Anderson (Ed.), Cognitive Skills and their Acquisition (pp. 1-55). Hillsdale, NJ: Earlbaum.

Thorburn, P. J., Jakku, E., Webster, A. J., \& Everingham, Y. L. (2011). Agricultural decision support systems facilitating co-learning: a case study on environmental impacts of sugarcane production. International Journal of Agricultural Sustainability, 9(2), 322-333. 


\section{Notes}

Note 1. Also, as a point of information we were not able to locate in the published literature any study that indicted that using DSS and the related creation of scenario re-parameterizations compromised sustainability.

Note 2. The specifics of this computation are: At baseline: 152,660 BSN RNs and 124,178 AD-D RNs. Each is expected to grow at $5 \%$ over the planning horizon yielding using the Excel function APP: $-\mathrm{FV}(.05,10,0,152,660)$ $\rightarrow$ 248,668 BSN RNs and -FV $(.05,10,0,124,178) \rightarrow 202,273$ BSN RNs. Now using Eq 8 (Kovner, Lee, Lusk, Katigbak, \&Selander, 2013) the additional number of BSN needed to be added to the workforce is $4 \times 202,273-$ $248,668=560,424$. Yes, this was the SAME set of baseline information that yielded in the SWAP context the need for 68,810 BSN RNs. This indicated the leverage multiplier effect of letting the AD-D workforce grow. Here on the order of 7 times more BSN RNs are needed.

Note 3. Another possibility of course is to modify the goals of the project. This possibility is not treated here as we are interested in trying to achieve the IOM 80/20 goal. Other goals should be considered as other projects and dealt with separately.

\section{Copyrights}

Copyright for this article is retained by the author(s), with first publication rights granted to the journal.

This is an open-access article distributed under the terms and conditions of the Creative Commons Attribution license (http://creativecommons.org/licenses/by/3.0/). 